\title{
Streptococcus faecalis Sex Pheromone (cAD1) Response: Evidence That the Peptide Inhibitor Excreted by pAD1-Containing Cells May Be Plasmid Determined
}

\author{
Don B. Clewell, ${ }^{*}$ Florence Y. An, ${ }^{*}$ MaSaAki Mori, $†$ YasuYoshi IKe, $\ddagger$ \\ AND AKINORI SUZUKI $\ddagger$ \\ ${ }^{*}$ Departments of Oral Biology and Microbiology/Immunology, Schools of Dentistry and Medicine, and the Dental \\ Research Institute, The University of Michigan, Ann Arbor, Michigan 48109-2007; \\ and + Department of Agricultural Chemistry, The University of Tokyo, Bunkyo-ku, Tokyo 113, and \\ $\ddagger$ Department of Microbiology. School of Medicine, Gunma University, Maebashi, Japan
}

Received June 4, 1986; revised September 3, 1986

\begin{abstract}
Streptococcus faecalis strains harboring the conjugative plasmid pAD1 excrete a small peptide, iAD1, which inhibits the sex pheromone cAD1. Studies making use of the host strain Streptococcus faecium 9790 , which normally does not excrete peptide pheromones, suggest that iAD1 may be determined directly by pADI. (1) 1987 Academic Press, Inc.
\end{abstract}

Streptococcus faecalis excretes peptide sex pheromones which induce a mating response in cells harboring certain conjugative plasmids (Dunny et al., 1978). The response involves the synthesis of a proteinaceous material, designated "aggregation substance," which facilitates the formation of mating aggregates upon random collision of donor and recipient cells (Yagi et al., 1983). Once a copy of the plasmid has been acquired by the recipient, activity of the related pheromone becomes undetectable in culture filtrates; the transconjugant, however, continues to express the activities of different pheromones specific for donors harboring different conjugative plasmids (Dunny et al., 1979; Clewell et al., 1982).

Donor cells have been found to excrete small peptides which act as competitive inhibitors of exogenous pheromone (Ike et al. 1983; Clewell et al. 1985). One possible purpose for the inhibitors is to slightly desensitize the donor cells in order to prevent a mating response to recipients that are too far away to result in random collisions. In such cases the low concentration of pheromone would be overcome by the inhibitor. It was proposed that the inhibitor represents a modified (inactivated) form of the pheromone (Ike et al. 1983).

The sex pheromones cPD1 (molecular weight 912) and cAD1 (molecular weight 818), which relate to the plasmids pPD1 ( $54.6 \mathrm{~kb}$; determines bacteriocin) and pAD1 ( $56.7 \mathrm{~kb}$; determines hemolysin-bacteriocin), respectively, have been isolated and sequenced (Suzuki et al., 1984; Mori et al., 1984). Both are hydrophobic octapeptides, and synthetic forms of each exhibited biological activity at concentrations as low as 5 $\times 10^{-11} \mathrm{M}$.

Recently the cAD1 inhibitor (iAD1) excreted by cells harboring a derivative of pAD1 has been isolated and sequenced (Mori et al., 1986); its structure, along with that of cAD1, is shown in Fig. 1. Clearly, iAD1 did not prove to be a modified form of CAD1; however, its homology with cAD1 (identity at four amino acid residue positions) points to the likely basis of its activity as a competitive inhibitor. The unique structure of the inhibitor raised the possibility that it may be plasmid determined. In addressing this question we reasoned that if $\mathrm{iAD} 1$ were encoded by pAD1, it may be possible to detect the inhibitor after introduction of the 
CAD1 H-LeU-PHE-SER-LeU-VAL-LEU-ALA-GLY-OH

IADI H-LEU-PHE-VAL-VAL-THR-LEE-VAL-GLLY -OH

FIG. 1. Amino acid sequences of the sex pheromone cADl and its inhibitor iAD1. The common residues are underlined. The molecular weight of $\mathrm{AADI}$ is 846 .

plasmid into a bacterium that does not normally excrete CAD1 (or iAD1). Streptococcus faecium 9790 has been found to be devoid of a number of sex pheromones (including cAD1) normally excreted by $S$. faecalis (Clewell et al., 1985). [One exception is cAM373; however, the role of this peptide as a true pheromone in this species has not been demonstrated (Clewell et al., 1985).] In the present study $S$. faecium 9790 was found to indeed excrete iAD1 when harboring, pAM714, a derivative of pAD1; these and related data are presented below.

The assay for cAD1 is based on the "clumping" response of cells harboring pAD1 (or a related derivative) when exposed to a culture filtrate of producer cells; and pheromone titers are obtained using a microtiter dilution method (serial twofold dilutions) (Dunny et al., 1979). Inhibitor activity is based on the ability to reduce the pheromone titer. In this case a pheromone-containing culture filtrate (FA2-2) was serially diluted through $50-\mu$ l aliquots of culture filtrate being tested for inhibitor activity prior to the addition of $50 \mu 1$ of responder cells (DS16). The number of units of inhibitor was taken as the pheromone titer in the absence of inhibitor (broth substituted for inhibitor) divided by the titer in the presence of inhibitor. In the case where chromatography was performed on $S$. faecium 9790RF (pAM714) material, the assay was according to Mori et al. (1986); and the amount of inhibitor was represented as $S$. faecalis iAD1 equivalents.

The plasmid pAM714 is a derivative of pAD1 (Ike and Clewell, 1984) containing an insert of the erythromycin resistance transposon Tn917. It was introduced into $S$. faecium 9790RF [which has mutational resistances to rifampin and fusidic acid (Clewell et al., 1985)] from $S$. faecalis OG1X(pAM714) (Ike and Clewell, 1984). Using an overnight filter mating procedure (Clewell $e t$ al., 1985), transfer to $S$. faecium occurred at approximately $10^{-6}$ per donor. The production of iAD1 by $S$. faecium 9790RF (pAM714) is shown in Fig. 2A. The activity increased with cell growth and leveled off as the culture entered stationary phase. The appearance of $\mathrm{AAD} 1$ was related to the presence of the plasmid, as no iAD1 activity could be detected in filtrates of a 9790 strain devoid of pAM714. That the structure of the iAD1 activity observed here was identical to that produced by $S$. faecalis carrying this plasmid

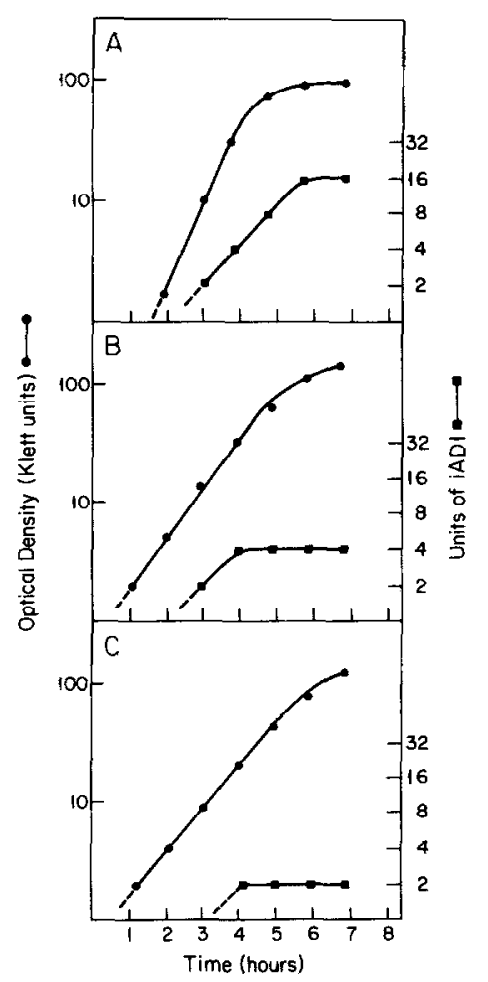

FIG. 2. Production of iAD1 by cells containing pAM714. (A) Streptococcus faecium 9790RF (pAM714). (B) Streptococcus faecalis JH2SS (pAM714). (C) Streptococcus faecalis Y11 (pAM714). The medium used was Todd Hewitt Broth (Difco) for $S$. faecium and N2GT (Dunny et al., 1979) for $S$. faecalis. Cell density was measured using a Klett-Summerson colorimeter (No. 54 filter). Culture volumes were $25 \mathrm{ml}$ and the activities were measured in filtrates of the sampled cultures. 


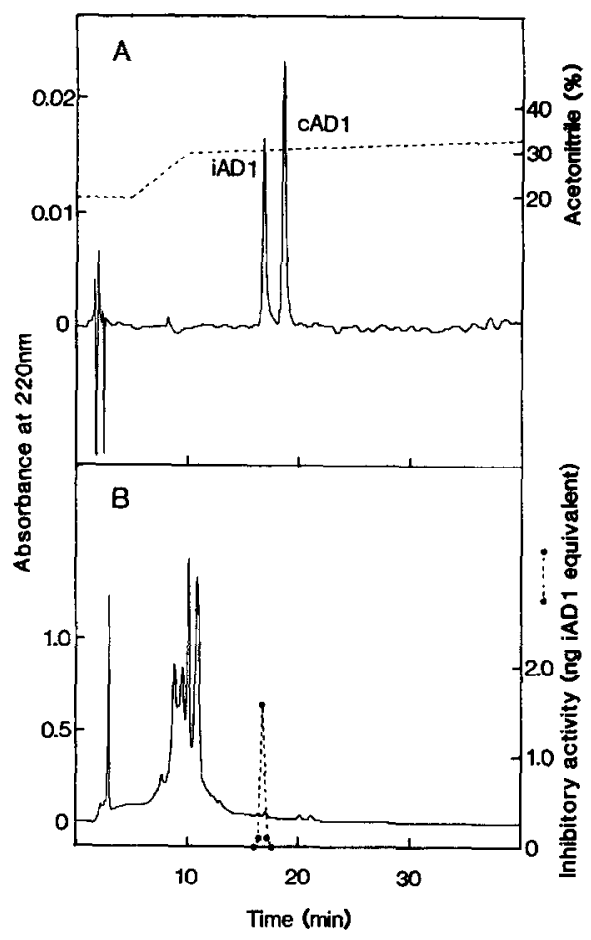

FIG. 3. Chromatography of (A) S. faecalis iAD1 and cAD1 and (B) partially purified $S$. faecium iADl-active fraction by reverse-phase HPLC. (A) Authentic $S$. faecalis iAD1 $(0.2 \mu \mathrm{g})$ and CAD1 $(0.3 \mu \mathrm{g})$ which had been previously synthesized (Mori et al., 1984, 1986) were coinjected and chromatographed on an SSC-ODS-262 column (Senshukagaku, $0.6 \times 10 \mathrm{~cm}$ ) with a gradient of 20 to $30 \%(5 \mathrm{~min})$ and 30 to $33 \%$ (30 min) acetonitrile in $10 \mathrm{mM}$ ammonium acetate at a flow rate of $1 \mathrm{ml} / \mathrm{min}$. (B) An overnight culture $(200 \mathrm{ml}$ containing $3.64 \mathrm{~g}$ of Oxoid Todd-Hewitt broth and $4 \mathrm{~g}$ of glucose) of $S$. faecium 9790RF (pAM714) was centrifuged and the supernatant was passed through an Amberlite XAD-7 column (Rohm and Haas, $5 \mathrm{ml}$ ). The activity absorbed was recovered with $30 \mathrm{ml}$ of $60 \%$ ethanol, and the eluate was threefold diluted and mixed with pyridine (final concentration 5\%). This solution was subjected to a QAESephadex A-25 column (Pharmacia, $5 \mathrm{ml}, \mathrm{Cl}^{-}$form) and eluted with $30 \mathrm{ml}$ of $0.1 \mathrm{M} \mathrm{NaCl}$. The eluate was then passed through an Amberlite XAD-2 column (Rohm and Haas, $5 \mathrm{ml}$ ), and the column was eluted with $30 \mathrm{ml}$ of $80 \%$ ethanol. The last eluate was evaporated and lyophilized, and the resulting powder was dissolved in $1 \mathrm{ml}$ of $50 \%$ acetonitrile. Twenty microliters of this solution (corresponding to $4 \mathrm{ml}$ of culture broth) was injected to the SSC-ODS-262 column and chromatographed under the same conditions as in $\mathrm{A}$. The eluate was fractionated with a fraction volume of $0.4 \mathrm{ml}$, and each fraction was bioassayed for $\mathrm{AAD} 1$ and CAD1 activity. No cAD1 activity was detected for any fractions. was strongly suggested from its characteristic retention time during fractionation by HPLC (Fig. 3).

We have also examined the production of iAD1 by $S$. faecalis Y11 (pAM714). Y11 is a mutant of $S$. faecalis JH2SS which is significantly reduced in the production of both cAD1 and cPD1 (Ike et al., 1983). It was generated using the conjugative transposon Tn 916 , and it produces only $3-4 \%$ the amount of pheromone excreted by its parent. As shown in Fig. 2C, Y11 (pAM714) produced levels of $\mathrm{iAD} 1$ corresponding to about half the level of that seen in Fig. 2B for JH2SS (pAM714). Although the amount of inhibitor produced by Y11 (pAM714) represents the lowest detectable level (by the assay used here), its integrity as $\mathrm{IADI}$ is supported by the similarity in the pattern of production; that is, in both the parent and mutant hosts, iAD1 activity leveled off in mid-log phase. [In the case of the parent, the difference in the pattern of production of iADl from that observed previously (Ike et al., 1983) appears to represent differences in the bacterial hosts as well as slight differences in the methodology used (B. White, personal communication).] Thus, despite the fact that the mutation in $Y 11$ reduced the yield of CAD1 to $3-4 \%$ that of the parent strain, the presence of pAM714 in this strain resulted in levels of $\mathrm{AAD} 1$ at least $50 \%$ that of the corresponding parent.

The data reported here are consistent with the view that pADI determines the production of $\mathrm{iAD} 1$. Although, the alternative possibility that pAD1 might activate the expression of a structural determinant located on the chromosome cannot be totally ruled out, such an occurrence seems unlikely. The absence of a cADl pheromone system in $S$. faecium 9790 implies there would be no functional basis for a host-encoded AAD1 in this strain. Independent of where the $\mathrm{AAD} 1$ structural locus resides, it is clear that in $S$. faecalis pAD1 affects the activity of CAD 1 by at least two means: the inactivation or repression of endogenous CADI and the pro- 
duction of an inhibitor of this peptide. Previous data (Ike et al., 1983) suggesting that donor strains excrete a form of CAD1 having a chemical addition (modification) that could be removed by phosphodiesterase II were misinterpreted, as there now appears to be a factor in N2GT broth that can be caused to induce clumping upon enzyme exposure (F. An and D. Clewell, unpublished). We believe, however, that a modification is a possible means by which endogenous CADI activity could be reduced in donor cells.

It remains likely that one of the roles of iAD1 is to desensitize donor cells to levels of exogenous CAD1 that are unproductively low (see above). The inhibitor may also act to prevent a self-induced mating response caused by low levels of endogenous CAD1 or different endogenous pheromones that may exhibit a low level of cross reactivity with cAD1. Although there is no evidence for cross reactivity between the pheromones cAD1 and cPD1 (Suzuki et al., 1984; Mori et al., 1984), it is conceivable that among yetunidentified activities, of which there may be many (Dunny et al., 1979; Clewell et al., $1982,1985)$, such phenomena could occur.

\section{ACKNOWLEDGMENTS}

This work was supported by U.S. Public Health Service Grants GM33956 and DE02731 and a Grant-in Aid for Scientific Research (NO. 60790127) from the Ministry of Education, Science and Culture of Japan. We thank our laboratory colleagues for helpful discussions and advice.

\section{REFERENCES}

Clewell, D., AN, F., White, B., and GawronBURKE, C. (1985). J. Bacteriol. 162, 1212-1220.

Clewell, D., YaGi, Y., IKE, Y., Craig, R., Brown, B., AND AN, F. (1982). In "Microbiology-1982" (D. Schlessinger, Ed.), pp. 97-100. Amer. Soc. for Microbiol., Washington, DC.

DuNNY, G., BRowN, B., AND Clewell, D. (1978). Proc. Natl. Acad. Sci. USA 75, 3479-3483.

DunNy, G., Craig, R., Carron, R., AND Clewell, D. (1979). Plasmid 2, 454-465.

IKE, Y., AND ClEWELL, D. (1984). J. Bacteriol. 158, 777-783.

IKe, Y., Craig, R., White, B., YaGi, Y., AND CleWell, D. (1983). Proc. Natl. Acad. Sci. USA. 80, 5369-5373.

Mori, M., Isogai, A., SaKagami Y., Fujino, M., KITADA, C., Clewell, D., AND Suzuki, A. (1986). Agric. Biol. Chem. 50, 539-541.

MORI, M., SAKaGami, Y., NARITA, M., IsOGaI, A., Fujino M., Kitada, C., Craig, R., Clewell, D., AND SUZUKI, A. (1984). FEBS Lett. 178, 97-100.

Suzuki, A., MORI, M., SAKaGami, Y., IsOgai, A., FuJino, M., Kitada, C., Craig, R., ANd Clewell, D. (1984). Science 226, 849-850.

YAGI, Y., KeSSleR, R., ShaW, J., Lopatin, D., AN, F., AND Clewell, D. (1983). J. Gen. Microbiol. 129, 1207-1215.

Communicated by Richard Novick 